FORMATION Formation emploi

Revue française de sciences sociales

142 | Avril-Juin 2018

Génération 2010 : diversité des parcours de réussite

\title{
Le travail salarié a-t-il un impact sur la réussite en première année de licence?
}

Does student employment have an impact on first year degree success?

Hat die bezahlte Arbeit einen Einfluss auf den Erfolg im ersten Jahr des

Bachelor-Studiums?

El trabajo asalariado ¿tiene impacto sobre el éxito en el primer año de

licenciatura?

\section{Sorho-Body Kady Marie-Danielle}

\section{(2) OpenEdition}

Journals

Édition électronique

URL : http://journals.openedition.org/formationemploi/5819

DOI : 10.4000/formationemploi.5819

ISSN : 2107-0946

Éditeur

La Documentation française

Édition imprimée

Date de publication : 23 août 2018

Pagination : $211-230$

ISSN : 0759-6340

Référence électronique

Sorho-Body Kady Marie-Danielle, «Le travail salarié a-t-il un impact sur la réussite en première année de licence? », Formation emploi [En ligne], 142 | Avril-Juin 2018, mis en ligne le 23 août 2020, consulté le 30 octobre 2020. URL : http://journals.openedition.org/formationemploi/5819; DOI : https:// doi.org/10.4000/formationemploi.5819 


\title{
Le travail salarié a-t-il un impact sur la réussite en première année de licence?
}

\author{
Sorho-Body Kady Marie-Danielle \\ Économiste, assistante à l'université Felix Houphouët-Boigny, Abidjan, Côte d'Ivoire, et \\ chercheur associé au Centre de recherche sur l'intégration économique et financière (CRIEF), \\ université de Poitiers - France
}

Résumé

Le travail salarié a-t-il un impact sur la réussite en première année de licence?

Cette étude vise à mesurer l'impact du travail salarié des étudiants sur leur réussite universitaire, en se focalisant sur les étudiants inscrits en première année de licence. L'emploi salarié et sa fréquence vont de pair avec une plus forte probabilité d'abandon des études et une plus faible probabilité de réussite. Toutefois, cet abandon n'est pas dû exclusivement à l'exercice d'une activité salarié hors cursus.

Mots clés : étudiant ; travail étudiant ; enseignement supérieur ; réussite scolaire ; abandon des études

Abstract

Does student employment have an impact on first year degree success?

This study aims to measure the impact of student employment on their academic achievement by focusing on students enrolled in the first year degree. Paid employment and its intensity are associated with a higher probability of dropping out of school and a lower probability of success. However, this abandonment is not due exclusively to the exercise of a salaried activity outside cursus.

Keywords: student ; student labour ; higher education ; success at school ; drop out Journal of Economic Literature : I 29 ; J 22 ; J 24

Traduction : Auteure. 


\section{Introduction}

En France, comme dans la plupart des pays de l'Union européenne, l'accès à l'université s'effectue sans aucune barrière ${ }^{1}$. Il suffit en effet de posséder le diplôme de fin d'études secondaires et que les possibilités d'accueil ne soient pas dépassées (Lafontaine \& Terwagne, 1993 ; Romainville, 1997 ; Michaut \& Romainville, 2012). Par conséquent, l'université accueille à la fois les étudiants ayant choisi leur filière de formation et ceux qui n'ont pas été retenus dans les filières sélectives.

L'absence de barrière à l'entrée est associée à un taux d'échec observé relativement important à l'issue de la première année (cf. Encadré 1) En France, le faible rendement, en termes de taux de réussite, de la première année universitaire, a également été dénoncé à maintes reprises ${ }^{2}$. Malgré les différentes politiques de lutte contre l'échec et les inégalités de réussite (réorientation en cours d'année, tutorat, Plan Réussite en Licence, etc.), la baisse de ce taux d'échec ne semble pas s'amorcer. Ainsi, on est tenté de penser que la sélection s'effectue à l'issue de la première année de licence.

\section{Encadré 1. Parcours et réussite des étudiants de premier cycle}

Le parcours des étudiants de $1^{\mathrm{er}}$ cycle est ponctué de réorientations et de redoublements : i) $50 \%$ des étudiants de L1 passent en L2, $25 \%$ redoublent et $25 \%$ se réorientent ou abandonnent leurs études. $70 \%$ des étudiants de L3 valident leur année (90\% pour les licences professionnelles) ; ii) $70 \%$ des étudiants inscrits en Institut universitaire de technologie (IUT) passent en année supérieure. Le taux de réussite en seconde année était de $90 \%$ en 2011. En Section de technicien supérieur (STS), le taux de passage en $2^{\text {ème }}$ année est de $85 \%$ et le taux de réussite est de $72 \%$ en 2011. Si l'on retient comme définition de l'échec en premier cycle le fait d'être sorti de l'enseignement supérieur sans diplôme, le taux d'échec en France est de l'ordre de 19\%, soit dix points de moins que la moyenne des pays de l'Organisation de coopération et de développement économiques (OCDE) (" Réussite et échec en $1^{\text {er }}$ cycle » Note d'information Enseignement Supérieur et Recherche 13.10, MESR-SIES, Novembre 2013).

Le faible taux de passage en L2 nous conduit à nous questionner sur les éventuelles causes de ce phénomène. Un des éléments fréquemment évoqué par les étudiants, pour justifier ce taux d'échec ou d'abandon à l'université, est l'emploi salarié exercé durant l'année universitaire.

Selon les dernières enquêtes de l'Observatoire national de la vie étudiante (OVE), près de $45 \%$ des étudiants déclarent exercer des activités salariées pendant l'année univer-

1. Il existe deux autres segments dans l'enseignement supérieur, aux caractéristiques antagonistes : i) un segment très sélectif (Grandes écoles, écoles d'ingénieurs, avec ou sans écoles préparatoires, CPGE, Santé) ; ii) un segment professionnalisant, sélectionnant un petit nombre d'étudiants, en vue d'une insertion professionnelle à court terme (formations courtes professionnelles en deux ou trois années d'études : STS, IUT Section de technicien supérieur et Institut universitaire de technologie - et écoles spécialisées) (Beaud, 2008 ; Coulon, 1997 ; Verley \& Zilloniz, 2010).

2. Pour une revue de la littérature, $c f$. Body, 2014. 
sitaire, dont $70 \%$ sont hors cursus. La part des étudiants qui exercent une activité rémunérée durant les études a légèrement baissé entre 2010 et 2013 (- 4 points de pourcentage), mais n’en reste pas moins assez élevée (Belghith, 2015).

L'emploi salarié exercé en cours de formation a-t-il une réelle incidence sur l'abandon en L1 ? En France, peu d'études empiriques se sont intéressées à cette question. On peut noter les travaux de Beffy \& al. (2009 ; 2013), à partir de l'enquête Emploi pour la période 1992-2002, ou ceux de Body \& al. (2014) sur une population d'étudiants inscrits à l'université de Poitiers en 2012. Ces études ne considèrent pas, pour des raisons de disponibilités des données, les étudiants inscrits en première année. La particularité de notre étude réside dans la mesure du lien existant entre l'emploi salarié et les résultats universitaires des étudiants de première année uniquement. Pour ce faire, nous mobilisons l'enquête Panel 1995 du ministère de l'Éducation nationale, qui concerne les élèves entrés en classe de sixième en 1995. Elle permet de repérer le cumul emploi-études, pour les étudiants s'inscrivant en première année de licence entre 2002 et 2004. En termes d'emploi, nous ne considérons que les activités salariées, hors cursus, occupées durant l'année universitaire. Les emplois de vacances ne sont pas retenus, n'étant pas, a priori, ou moins directement en concurrence avec le temps studieux.

Concernant les résultats universitaires, trois situations sont envisagées, à savoir l'abandon en cours d'année et la validation ou non de l'année. Plus précisément, un étudiant qui abandonne est un étudiant qui ne s'est pas présenté aux examens de L1. Ainsi, la définition de l'abandon retenue ici englobe aussi bien les étudiants qui arrêtent définitivement leurs études que ceux qui reprennent leurs études l'année suivante (réorientation ou redoublement). Nous considérons que l'année universitaire n'est pas validée si l'étudiant n'a pas obtenu, sur l'année, la moyenne aux examens (y compris la validation d'un semestre); dans le cas contraire, l'année est validée (l'étudiant peut, s'il le souhaite, passer en L2).

Dans cette analyse, à l'instar de Beffy \& al. (op. cit.) ou Body \& al. (op. cit.), nous proposons de repérer les effets des caractéristiques de l'emploi salarié sur la réussite universitaire ou l'abandon des études, tout en prenant en compte la nature endogène de la décision de travailler durant les études.

Les résultats obtenus montrent que l'emploi salarié va de pair avec une plus faible probabilité de valider l'année et une plus forte probabilité d'abandonner les études en cours d'année.

L'originalité de ce travail réside dans l'étude du lien existant entre emploi étudiant et abandon en cours de formation. Dans la partie 1, nous rappelons quelques résultats empiriques liés au travail salarié étudiant, à l'échec et l'abandon en première année de licence. La partie 2 présente les données utilisées issues de l'enquête " Panel 1995 » du ministère de l'Éducation nationale. La partie 3 présente les modélisations retenues. Afin de tenir compte du caractère endogène de la décision d'exercer une activité salariale, 
nous y développerons la technique des variables instrumentales. Les résultats sont quant à eux présentés dans la partie 4 et il s'avère que l'emploi salarié diminue fortement les chances de succès et augmente aussi les risques d'abandon des études.

\section{L'échec en L1 : le rôle des déterminants sociaux, scolaires ou cognitifs}

Certains travaux en sciences de l'éducation se sont intéressés à l'abandon et à la réussite en L1. Le fort taux d'échec observé peut s'expliquer, en partie, par des déterminants sociaux, scolaires ou encore cognitifs. Les études mettent en évidence un effet de la série du baccalauréat, de la mention et du retard scolaire. Ainsi, être titulaire d'un baccalauréat professionnel ou technologique, ne pas avoir obtenu son baccalauréat avec mention ou encore s'inscrire à l'université avec un retard scolaire augmentent la probabilité d'échec (Beaupère \& Boudesseul, 2009 ; Morlaix \& Suchaut, 2015 ; Duguet \& al., 2016).

Parallèlement, des indicateurs spécifiques de compétences mesurant l'aptitude cognitive (mémoire de l'étudiant, vitesse de traitement de l'information et raisonnement logique) et les performances académiques (niveau de compréhension à l'écrit) des étudiants jouent un rôle positif limité sur les notes (Morlaix \& Suchaut, op. cit.)).

Surtout, la motivation pour les études peut jouer un rôle important sur le taux d'échec. D’une part, pour certains étudiants, l'université ne constitue pas le premier choix d'études supérieures. On retrouve ici les étudiants n'ayant pas été sélectionnés dans la formation désirée. Ils sont donc moins motivés et s'investissent moins dans les programmes universitaires. D'autre part, le manque de motivation peut être associé à un manque de vocation. Les étudiants n'ayant pas de projet d'avenir précis vont sélectionner leur orientation par tâtonnement. Les étudiants ayant un projet professionnel ont une moindre probabilité d'échec (Morlaix \& Suchaut, op. cit.). De manière générale, la motivation des étudiants semble avoir un effet direct, mais aussi un effet indirect sur la réussite.

Certains étudiants s'inscrivent à l'université afin d'améliorer leurs connaissances dans des matières spécifiques nécessaires à l'obtention de leur concours ou plus simplement, comme c'est le cas pour les écoles de travailleurs sociaux, en attendant de vieillir un peu pour paraître plus " matures" (Bodin, 2009). Pour eux, l'université constitue un tremplin vers des formations futures, car pour beaucoup d'écoles spécialisées, il n'existe pas ou peu de classes préparatoires. C'est généralement le cas de nombreux étudiants inscrits en sciences du vivant, psychologie, sociologie ou en sport afin d'accéder aux écoles du social ou du paramédical (Bodin \& Millet, 2012). 
Les étudiants peuvent ne pas avoir correctement perçu les exigences de leur " métier » d'étudiant et rencontrent un problème d'adaptation. Pour eux, la compréhension et l'incorporation des codes de la culture universitaire ne se font pas ou se font mal (Coulon, op. cit.). Il apparaît que cette adaptation est étroitement liée aux caractéristiques socio-économiques de l'étudiant et à sa trajectoire scolaire passée.

Toutefois, indépendant du passé scolaire, mais non de l'origine sociale, l'exercice d'une activité rémunérée constitue un facteur d'échec immédiat (Bisseret, 1968). En effet, outre les raisons évoquées ci-dessus, l'obligation, pour des étudiants, de travailler durant les études pour des motifs financiers, est souvent désignée comme une cause d'échec et d'abandon des études (Ehrenberg \& Sherman, 1987 ; McNeal Jr., 1997 ; Montmarquette \& al., 2007 ; Brint \& Cantwell, 2010 ; Beffy \& al., op. cit. ; Duguet \& al., op. cit.). Ces activités concurrentes au temps d'études réduiraient l'effort studieux et donc la réussite étudiante.

Dans cet article, nous approfondissons le lien existant entre emploi salarié et résultats universitaires pour des étudiants de L1. Selon les données Eurostudent ${ }^{3}$, avec $50 \%$ d'étudiants qui travaillent, la France se caractérise par un grand nombre d'emplois occasionnels peu ou pas liés aux études ${ }^{4}$. Bien que la principale raison de cette pratique soit financière, on pourrait supposer qu'elle est aussi stratégique. En effet, l'exercice d'un emploi salarié, pendant les études, pourrait être associé à l'acquisition d'une expérience professionnelle pouvant constituer un bon signalement à l'issue de la formation.

\section{- Mobiliser le panel 1995 d'élèves de 6ème}

Nous utilisons la base " Panel 1995 " du ministère de l'Éducation nationale. Elle se compose d'un échantillon d'élèves scolarisés en classe de sixième pendant la rentrée scolaire 1995-1996, dans un établissement public ou privé de la métropole. Ces élèves ont été suivis jusqu'en 2007. Parmi les 17830 élèves de $6^{\mathrm{ème}}$ interrogés en 1995, 11681 ont suivi une classe de seconde, 10779 sont allés jusqu'en classe de terminale, 10085 ont passé les épreuves du baccalauréat et 9245 l'ont validé.

3. Ces données montrent la diversité des situations de cumul emploi-études en Europe : près de deux étudiants danois sur trois travaillent, mais seulement un étudiant sur trois travaille dans les pays du sud de l'Europe, comme l'Espagne et l'Italie (Belghith \& Vourc'h, 2011).

4. Aux États-Unis, plus de $80 \%$ des étudiants du premier cycle exercent un emploi salarié hors cursus (Baum, 2010 ; Scott-Clayton, 2012). 
Seulement 7472 élèves poursuivent leurs études post-baccalauréat. Nous considérerons les $2199^{5}$ étudiants inscrits, au cours de leur première année d'études supérieures, dans une université française, hors IUT et études médicales.

Pour chaque étudiant, nous observons ses caractéristiques individuelles et familiales (sexe, âge, logement occupé durant l'année universitaire, pays de naissance des parents), ses caractéristiques scolaires passées (redoublement et/ou retard scolaire, type de baccalauréat obtenu, mention au baccalauréat), ses caractéristiques universitaires (filière suivie, localisation de l'université et choix d'orientation souhaité à l'issue du baccalauréat, cette information permettant de voir si la L1 suivie correspond au premier choix de l'étudiant). Des caractéristiques plus subjectives sont aussi disponibles (diplôme de plus haut niveau souhaité, vision de l'avenir, problèmes personnels $\left.{ }^{6}\right)$.

Enfin, une variable de confiance en soi a été construite à partir d'un indice composite calculé à l'aide d'une analyse en composantes multiples. Elle a été construite grâce aux réponses à des questions sur l'estime de soi et la sociabilisation ${ }^{7}$. À partir de cet indice, les $10 \%$ ayant le moins confiance en eux et les $10 \%$ ayant le plus confiance en eux ont été introduits dans les modélisations effectuées ici.

Près de $40 \%$ des étudiants de l'échantillon déclarent occuper un emploi durant l'année universitaire. 25,8 \% des étudiants occupent un emploi occasionnel (activités rémunérées exercées de manière ponctuelle) et 12,8\% un emploi régulier (tout emploi avec contrat de travail - CDI ou CDD - contrat à durée indéterminée et contrat à durée déterminée - exercé de manière continue) au cours de la $\mathrm{L}^{8}$ ( $c f$. Tableau 1). La proportion d'étudiants ayant abandonné leurs études ${ }^{9}$ est d'environ $16 \%$. Notons qu'avec

5. Parmi les 7472 étudiants qui poursuivent leurs études après le baccalauréat, 807 sont inscrits en Classe préparatoire aux grandes écoles, 3050 en première année d'université, 1814 en BTS, 887 en IUT et 914 dans une autre formation. Pour l'université, ont été supprimés les étudiants n’ayant pas répondu aux questions clés de notre analyse, ainsi que ceux inscrits dans un diplôme universitaire (DU), un diplôme d'études universitaires scientifiques et techniques (DEUST) ou en filière santé car, pour la cohésion des interprétations, nous faisons le choix d'évaluer exclusivement des étudiants ayant le même mode d'évaluation.

6. Les étudiants répondent aux questions suivantes : "Comment voyez-vous votre avenir?" et "Avez-vous des problèmes personnels autres que financiers?".

7. Les élèves répondent à huit affirmations du type "Je suis très apprécié(e) de mes copains ", "Je suis souvent content(e) de moi ", "J'ai beaucoup de copains". Quatre réponses étaient possibles : pas du tout d'accord, pas vraiment d'accord, plutôt d'accord, tout à fait d'accord.

8. Le volume horaire travaillé, pour les étudiants de L1, est d'environ huit heures hebdomaires (Froment, 2012). Dans l'enquête, la durée hebdomadaire de travail est observée de façon dichotomique (plus ou moins de quinze heures) seulement pour les emplois réguliers. Nous avons préféré ne pas utiliser l'information sur les heures et mobiliser la fréquence des emplois : occasionnels et réguliers. Les aides financières correspondent aux aides de la famille, aux bourses d'études et autres aides de l'État.

9. Ce taux est peut-être sous-évalué dans la mesure où les questions relatives aux résultats académiques de la L1 ont été posées au cours de l'enquête réalisée l'année universitaire suivante. Par conséquent, les étudiants n'ayant pas répondu à cette enquête (phénomène d'attrition) ont été supprimés de l'analyse et il n'est pas 
plus de $34 \%$, cette proportion est significativement plus élevée pour les étudiants occupant un emploi salarié régulier durant l'année universitaire. Que les étudiants occupent ou non un emploi, le taux d'échec ou de validation partielle est comparable (autour de $30 \%$ ). 53,4\% des étudiants valident leur année. Ce taux de réussite est de $56,4 \%$ pour les étudiants non salariés, 55,9\% pour les travailleurs occasionnels et de $34 \%$ pour les travailleurs réguliers.

Ces statistiques descriptives laissent présager que l'exercice régulier d'un emploi salarié hors cursus augmente le risque d'abandon des études et que les emplois exercés de manière occasionnelle n'ont pas d'impact négatif sur la réussite universitaire.

Tableau 1. Statistiques descriptives sur le travail salarié et les résultats universitaires

\begin{tabular}{|c|c|c|c|c|c|}
\hline & & & & riés & \\
\hline & & Ensemble & $\begin{array}{c}\text { Emploi } \\
\text { occasionnel }\end{array}$ & Emploi régulier & Non-salariés \\
\hline & Effectif & 2199 & 567 & 282 & 1350 \\
\hline & $\%$ & 100 & 25,78 & 12,82 & 61,39 \\
\hline & Abandon & 15,92 & 14,99 & 34,04 & 12,52 \\
\hline Résultats universitaires (\%) & Année non validée & 30,7 & 29,10 & 31,91 & 31,11 \\
\hline & Année validée & 53,39 & 55,91 & 34,04 & 56,37 \\
\hline
\end{tabular}

Note de lecture : parmi l'ensemble des étudiants enquêtés, $15,9 \%$ abandonnent leurs études, 30,7 \% échouent et $53,4 \%$ réussissent totalement leurs examens.

Les emplois occasionnels sont ceux exercés de façon ponctuelle au cours de l'année universitaire, généralement sans contrat de travail ; les emplois réguliers sont des emplois permanents exercés, la plupart du temps, sous contrat de travail et avec une durée bien déterminée.

Source : Panel 1995, ministère de l'Éducation nationale.

\section{Modéliser l'interaction entre emploi 3 et résultats de la première année}

L'emploi salarié va être modélisé de deux façons, selon que l'étudiant travaille ou non, d'une part, et selon la régularité de l'emploi exercé (régulier ou occasionnel), d'autre part.

En outre, le fait d'occuper un emploi salarié durant l'année universitaire résulte de différentes variables observées ou non observées qui peuvent affecter la probabilité d'abandon ou de réussite de l'année. On peut penser aux caractéristiques scolaires (passées ou présentes) et à l'environnement social de l'étudiant qui sont généralement

possible de corriger ce biais car nous n'avons aucune information sur ces étudiants. 
des caractéristiques observables. Mais des variables non observables telles que la motivation ou encore les compétences et les aptitudes de l'étudiant ne sont pas toujours connues. Ces éléments peuvent affecter de la même manière ou de manière opposée la décision de travailler et le parcours académique.

Lexistence de variables explicatives non observées pertinentes nous a conduit à estimer des modèles de type Probit à deux équations simultanées conformément à d'autres travaux réalisés sur ce thème (Beffy \& al., op. cit.; Body, op. cit. ; Body\& al., op. cit. $)^{10}$. Afin d'identifier l'effet du travail salarié, nous utilisons la technique des variables instrumentales. Un instrument est une variable qui explique directement et significativement la variable suspectée d'être endogène (le travail salarié), mais qui n'explique pas directement la variable dépendante (l'abandon ou les résultats à la fin de l'année universitaire). Les instruments retenus dans l'analyse sont le logement occupé par l'étudiant et les variables de ressources (i.e. l'acquisition d'une bourse et l'obtention d'une aide financière familiale $)^{11}$. Ces deux variables, qui relèvent de l'autonomie de l'étudiant, sont liées à l'exercice d'un emploi salarié, mais ne semblent pas avoir d'effet direct sur la réussite universitaire, comme l'indiquent Kalenkoski \& Pabilonia (2009) à partir de données américaines, ou encore Gruel (2002) et Morlaix \& Suchaut (2015) sur des données françaises. Les variables explicatives introduites dans les deux équations sont associées aux caractéristiques socio-économiques, scolaires, universitaires et subjectives de l'étudiant. Les paramètres des deux équations et le coefficient de corrélation entre les deux équations sont estimés en même temps, à partir d'une fonction de vraisemblance.

Nous allons considérer trois modèles. Le premier modèle traitera simultanément la probabilité d'exercer un emploi salarié hors cursus en cours de formation et celle associée à l'abandon des études. Cette modélisation permettra de mesurer un effet causal net de l'emploi salarié sur l'abandon. La seconde modélisation estimera, quant à elle, de manière simultanée, la probabilité de travailler durant les études et celle associée aux résultats universitaires. La variable de résultats universitaires prend trois modalités ordonnées afin de hiérarchiser les situations de réussite : l'étudiant a abandonné son année, il n’a pas validé son année et il a validé son année. La troisième modélisation

10. Une solution alternative aurait pu consister à utiliser les méthodes d'estimation de type matching et score de propension et à réaliser ensuite une analyse de sensibilité, comme celle proposée par Ichino \& al. (2008) pour vérifier si l'influence des facteurs inobservés biaise les estimations. La robustesse de ces méthodes ayant été démontrée seulement lorsque les variables de traitement (ici l'emploi salarié) et les variables d'intérêt (les résultats universitaires) n’ont que deux modalités, nous avons préféré utiliser une méthode de variables instrumentales.

11. Une variable macro-économique caractérisant la situation sur le marché du travail, comme le taux de chômage des jeune, pourrait être un bon instrument ; malheureusement, étant donné que nous travaillons avec des données en coupe, cette variable aurait peu de variabilité et serait fortement corrélée avec la zone géographique. 
proposée est une prolongation de la deuxième. Nous introduisons ici une hétérogénéité de l'activité salariale en tenant compte de la régularité de l'emploi occupé. En effet, selon sa fréquence, le travail salarié empiète sur le temps de loisirs de l'étudiant et/ou sur son temps de révision et pourrait donc avoir des conséquences différentes sur ses résultats universitaires. Pour tenir compte du type d'emploi salarié exercé, la première équation du modèle sera légèrement modifiée et prendra trois modalités ordonnées (l'étudiant n'occupe pas d'emploi ; l'étudiant exerce un emploi salarié de manière occasionnelle ; l'étudiant exerce un emploi salarié de façon régulière). Les résultats relatifs à ces modélisations sont présentés dans la partie suivante.

\section{Les effets d'un emploi salarié exercé en cours de formation}

Les résultats sont interprétés toutes choses égales par ailleurs. Cette partie présente les résultats des trois modélisations effectuées. La première modélisation traite de l'impact de l'exercice d'un emploi salarié sur l'abandon de la formation, la seconde de l'impact du travail salarié sur les résultats universitaires et enfin la troisième tient compte des différentes fréquences de l'emploi salarié (pas d'emploi ; emploi occasionnel ; emploi régulier).

L’endogénéité de l'emploi salarié est vérifiée, puisque les coefficients de corrélation entre les termes d'erreurs associés à l'emploi salarié et à l'abandon ou aux résultats universitaires sont significatifs. Les résultats des deux premières estimations ${ }^{12}$ sont présentés dans le Tableau 2 et ceux inhérents à la fréquence de l'emploi le sont dans le Tableau 3.

12. Pour l'estimation du second modèle (résultats universitaires), les résultats des estimations associés à l'équation du travail ne sont pas reportés car ils sont très proches de ceux obtenus pour le premier modèle (abandon en première année de licence). Ils sont disponibles sur demande. 
Tableau 2. Emploi salarié, résultats universitaires et abandon des études

\begin{tabular}{|c|c|c|c|}
\hline & $\begin{array}{c}\text { Coefficient } \\
\text { Travail }\end{array}$ & $\begin{array}{c}\text { Coefficient } \\
\text { abandon }\end{array}$ & $\begin{array}{c}\text { Coefficient } \\
\text { résultats }\end{array}$ \\
\hline Constante & $-0,551$ & $-1,843$ & 1,895 \\
\hline \multicolumn{4}{|l|}{ Caractéristiques individuelles et familiales } \\
\hline Sexe: homme & $-0,227$ & 0,232 & $-0,210$ \\
\hline Au moins 1 parent a fait des études sup. & $0,050 \mathrm{~N}$ & $-0,106 \mathrm{~N}$ & 0,141 \\
\hline \multicolumn{4}{|l|}{ Oritine des parents } \\
\hline \multicolumn{4}{|l|}{ Parents nés en France (ref) } \\
\hline Parents nés en Europe & $-0,153 \mathrm{~N}$ & $-0,075 \mathrm{~N}$ & $0,010 \mathrm{~N}$ \\
\hline Un parent est né hors d'Europe & $-0,032 \mathrm{~N}$ & $0,070 \mathrm{~N}$ & $-0,064 \mathrm{~N}$ \\
\hline Parents nés hors d'Europe & $-0,098 \mathrm{~N}$ & $-0,026 \mathrm{~N}$ & $-0,057 \mathrm{~N}$ \\
\hline \multicolumn{4}{|l|}{ Conditions de vie } \\
\hline Problème personnel : oui & 0,145 & 0,243 & $-0,291$ \\
\hline \multicolumn{4}{|l|}{ Confiance en soi } \\
\hline Très bonne & $0,186^{*}$ & $-0,083 \mathrm{~N}$ & $-0,081 \mathrm{~N}$ \\
\hline \multicolumn{4}{|l|}{ Normale (ref) } \\
\hline Très mauvaise & $-0,068 \mathrm{~N}$ & $-0,072 \mathrm{~N}$ & $0,144 \mathrm{~N}$ \\
\hline \multicolumn{4}{|l|}{ Logement occupé par l'étudiant } \\
\hline Chez les parents ou de la famille & 0,435 & & \\
\hline En logement individuel & 0,222 & & \\
\hline \multicolumn{4}{|l|}{ En résidence universitaire (ref) } \\
\hline Titulaire d'une bourse d'études & $-0,411$ & & \\
\hline Bénéficie d'une aide financière & $-0,130$ & & \\
\hline \multicolumn{4}{|l|}{ Scolarité passée } \\
\hline \multicolumn{4}{|l|}{ Retard scolaire à l'entrée à l'université } \\
\hline Avance & $-0,230 \mathrm{~N}$ & $0,076 \mathrm{~N}$ & $-0,109 \mathrm{~N}$ \\
\hline \multicolumn{4}{|l|}{ Normal (ref) } \\
\hline Retard (sans redoublement) & $0,132 \mathrm{~N}$ & $-0,457$ & $0,119 \mathrm{~N}$ \\
\hline Retard (avec redoublement) & $0,074 \mathrm{~N}$ & - & - \\
\hline Redoublement au primaire & - & $0,040 \mathrm{~N}$ & $-0,089 \mathrm{~N}$ \\
\hline Redoublement au lycée & - & 0,284 & $-0,355$ \\
\hline Autre type de redoublement & - & $0,186^{*}$ & $-0,265$ \\
\hline Obtention d'une mention au baccalauréat & $-0,042 \mathrm{~N}$ & $-0,302$ & 0,651 \\
\hline \multicolumn{4}{|l|}{ Type de baccalauréat obtenu } \\
\hline \multicolumn{4}{|l|}{ Scientifique (ref) } \\
\hline Economie & $0,132 \mathrm{~N}$ & $0,047 \mathrm{~N}$ & $-0,186$ \\
\hline Littéraire & $0,084 \mathrm{~N}$ & $0,224^{*}$ & $-0,469$ \\
\hline Technologique & $0,145 \mathrm{~N}$ & $0,180 \mathrm{~N}$ & $-0,658$ \\
\hline Professionnel & $0,117 \mathrm{~N}$ & $0,277 \mathrm{~N}$ & $-0,800$ \\
\hline \multicolumn{4}{|l|}{ Scolarité à l'université } \\
\hline \multicolumn{4}{|l|}{ Premier choix d'orientation après le bac } \\
\hline Licence 1 suivie (ref) & & & \\
\hline
\end{tabular}




\begin{tabular}{|c|c|c|c|}
\hline & $\begin{array}{c}\text { Coefficient } \\
\text { Travail }\end{array}$ & $\begin{array}{l}\text { Coefficient } \\
\text { abandon }\end{array}$ & $\begin{array}{l}\text { Coefficient } \\
\text { résultats }\end{array}$ \\
\hline BTS-IUT & 0,263 & 0,281 & $-0,244$ \\
\hline Classe préparatoire & $0,200 \mathrm{~N}$ & $-0,272 \mathrm{~N}$ & $0,117 \mathrm{~N}$ \\
\hline Autre L1 que celle suivie & $0,062 \mathrm{~N}$ & 0,665 & $-0,611$ \\
\hline Autre choix & $0,014 \mathrm{~N}$ & 0,639 & $-0,538$ \\
\hline \multicolumn{4}{|l|}{ Spécialité de formation suivie } \\
\hline \multicolumn{4}{|l|}{ Droit-économie-gestion (ref) } \\
\hline Sciences humaines-lettres & $-0,190^{*}$ & $-0,041 \mathrm{~N}$ & 0,226 \\
\hline Sciences fondamentales & $0,130^{*}$ & $0,113 \mathrm{~N}$ & $-0,212$ \\
\hline Autres spécialités & $0,043 \mathrm{~N}$ & $-0,082 \mathrm{~N}$ & $0,089 \mathrm{~N}$ \\
\hline \multicolumn{4}{|l|}{ Lieu d'études } \\
\hline Région parisienne & 0,256 & $-0,123 \mathrm{~N}$ & 0,155 \\
\hline Toulouse & $0,098 \mathrm{~N}$ & $0,034 \mathrm{~N}$ & $0,076 \mathrm{~N}$ \\
\hline Marseille & $-0,093 \mathrm{~N}$ & $0,215 \mathrm{~N}$ & $-0,167 \mathrm{~N}$ \\
\hline Lyon & 0,438 & $-0,302^{*}$ & $0,174 \mathrm{~N}$ \\
\hline Rennes & $0,167 \mathrm{~N}$ & $-0,028 \mathrm{~N}$ & $0,162 \mathrm{~N}$ \\
\hline Lille & $-0,222 \mathrm{~N}$ & 0,346 & $-0,372$ \\
\hline Bordeaux & 0,296 & $-0,038 \mathrm{~N}$ & $-0,093 \mathrm{~N}$ \\
\hline \multicolumn{4}{|l|}{ Autres (ref) } \\
\hline \multicolumn{4}{|l|}{ Avenir après l'université } \\
\hline \multicolumn{4}{|l|}{ Diplôme de plus haut niveau souhaité } \\
\hline $\mathrm{Bac}+2$ & $-0,038 \mathrm{~N}$ & 0,639 & $-0,687$ \\
\hline Licence & $-0,045 \mathrm{~N}$ & 0,307 & $-0,285$ \\
\hline \multicolumn{4}{|l|}{ Master (ref) } \\
\hline Thèse & $0,034 \mathrm{~N}$ & $-0,059 \mathrm{~N}$ & $0,099 \mathrm{~N}$ \\
\hline \multicolumn{4}{|l|}{ Vision de l'avenir } \\
\hline \multicolumn{4}{|l|}{ Bonne (ref) } \\
\hline Mauvaise & $0,005 \mathrm{~N}$ & $-0,104 \mathrm{~N}$ & $-0,036 \mathrm{~N}$ \\
\hline Ne sait pas & $-0,214$ & $-0,016 \mathrm{~N}$ & $-0,005 \mathrm{~N}$ \\
\hline Occupe un emploi salarié durant l'année & & 1,412 & $-0,855$ \\
\hline $\mathrm{S}_{2}$ & & & 1,081 \\
\hline$\sigma_{\mathrm{FY}}$ & & $-0,691$ & 0,391 \\
\hline
\end{tabular}

Note de lecture : Toutes choses égales par ailleurs, les hommes ont une probabilité plus faible d'occuper un emploi salarié durant leurs études que les femmes. Ils ont également une probabilité plus forte d'abandonner leurs études et plus faible de réussir leurs examens que les femmes.

Etant donné la construction d'un modèle probit ordonné, les coefficients associés au modèle simple (abandon) et au modèle probit ordonné incluant les résultats auront des signes opposés. Pour le modèle ordonné, un coefficient positif (resp. négatif) et significatif s'interprète de la façon suivante : la variable associée à ce coefficient augmente (resp. diminue) la probabilité de réussite et diminue (resp. augmente) la probabilité d'abandon.

Légende : pour chaque colonne, le chiffre présenté est le paramètre estimé significatif ( ${ }^{*}$ significatif à $10 \%$, N : modalités non significatives).

Source : Panel 1995, ministère de l'Éducation nationale. 
Les interprétations des différents résultats présentés dans le Tableau $\mathbf{2}$ se feront dans les deux points suivants. La point 4.1 traitera de l'impact de l'emploi salarié sur l'abandon en cours de formation et le point 4.2 des effets du travail salarié et de sa fréquence sur les résultats universitaires.

\subsection{L'emploi salarié, ses déterminants et son effet sur l'abandon}

\subsubsection{Les déterminants de l'emploi salarié}

L'estimation de la première équation est présentée dans la première colonne du Tableau 2. Il s'agit d'identifier les déterminants de l'emploi salarié hors cursus exercé en cours de formation.

Parmi les caractéristiques individuelles et familiales, seul le sexe a un impact significatif sur la probabilité d'occuper un emploi salarié. Ainsi, les hommes ont une probabilité plus faible que les femmes d'occuper un emploi salarié durant les études. Deux arguments peuvent être avancés pour expliquer ce résultat. D’une part, les emplois de services, abondamment proposés aux étudiants, sont occupés plus fréquemment par les femmes (durant ou après les études), spécialement ceux qui impliquent un contact direct avec le public (Hochschild, 1983 ; Wharton, 2004). D'autre part, la volonté des étudiantes à être autonomes est plus forte. Un effet de la CSP (catégorie socio-professionnelle) des parents est mis en évidence, puisque les enfants de cadres supérieurs et dans une moindre mesure, de cadres moyens ont un accès plus fréquent à un emploi durant les études, comme le confirment Body (2014) ; Body \& al. (2014); ou encore Beffy \& al. (2009; 2013). Nous avons approximé la CSP par le fait que l'un des parents au moins a suivi des études supérieures. Cette variable n'affecte pas la probabilité d'occuper un emploi salarié ${ }^{13}$. L'effet de la CSP des parents sur l'accès à l'emploi peut être vu comme un effet de réseau qui ne semble pas être capté par le niveau d'études des parents.

Le parcours scolaire passé, résumé par le retard scolaire, le baccalauréat préparé ou l'obtention d'une mention, n'impacte pas la probabilité de travailler.

En revanche, les caractéristiques liées à l'université, au choix d'orientation et à la filière suivie sont discriminantes. Les étudiants dont le premier choix était de s'orienter en BTS ou en IUT ont une plus forte probabilité de travailler. Inscrits à l'université par défaut, peut-être ont-ils un intérêt et/ou une motivation moindres pour la formation suivie, qu'ils n'ont pas choisie. Aussi, la filière d'inscription impacte aussi la décision de travailler, puisque les étudiants inscrits en sciences fondamentales ont plus de chances

13. La CSP des parents est observée lors d'une enquête menée auprès des familles en 1998. Etant donné leur date d'entrée au collège (1995), la première vague d'élèves entrés à l'université se situe en 2002. La CSP observée en 1998 est donc un peu ancienne. Cette information a été testée, mais les coefficients ne sont pas significatifs. 
d'exercer une activité salariée durant leurs études que ceux inscrits en droit, économie et gestion, tandis que ceux inscrits en sciences humaines et lettres ont moins de chances de travailler.

L'octroi d'une bourse diminue la probabilité d'occuper un emploi salarié. Ce résultat n'est pas surprenant car les bourses de l'État limitent ou même parfois interdisent la possibilité de cumuler emploi et études. De plus, recevoir une aide financière des parents diminue la probabilité de travailler durant les études. Autrement dit, à origine sociale équivalente, occuper un emploi durant les études répond à un besoin de revenu qui permet de compenser l'absence d'aide financière de la famille. L'aide parentale ne semble être bénéfique qu'aux plus privilégiés, représentés par les étudiants issus de milieux sociaux favorisés et/ou qui suivent des études longues ou sélectives (Galland, 2016).

Vivre en logement individuel ou encore vivre chez ses parents ou un autre membre de sa famille augmente la probabilité d'occuper un emploi salarié durant les études. Ces résultats sont conformes à la littérature. Les logements en résidence universitaire étant généralement réservés aux étudiants boursiers, l'emploi salarié répond donc plus à un besoin d'autonomisation financière. Cependant, les étudiants résidant à Paris, Lyon ou Bordeaux ont plus de chances d'occuper un emploi salarié. Ce résultat peut s'expliquer par la situation du marché du travail local dans ces villes.

Enfin, les caractéristiques subjectives structurent également les chances d'exercer une activité. En effet, avoir des " problèmes personnels autres que financiers » (cf. note 6) ou une très bonne confiance en soi sont des facteurs qui augmentent la probabilité d'occuper un emploi salarié. Inversement, n'avoir aucune idée quant à son avenir (professionnel ou pas) diminue la probabilité d'occuper un emploi salarié durant l'année universitaire.

\subsubsection{Exercer un emploi salarié : quel impact sur l'abandon des études ?}

Les résultats de cette estimation sont fournis dans la deuxième colonne du Tableau $2^{14}$. Il est nécessaire d'estimer simultanément les probabilités d'exercer un emploi salarié en cours de formation et celle d'abandonner ses études, car le coefficient de corrélation est significatif.

L'exercice d'un emploi salarié en cours de formation augmente de manière significative la probabilité d'abandonner ses études. Le signe négatif du coefficient de corrélation vient atténuer légèrement cet effet. Cela suggère en effet que les facteurs inobservés (tels que le faible intérêt pour les études ou le désir d'indépendance financière) favorables à l'exercice d'un emploi salarié encouragent aussi la décision d'abandon des études.

14. Ils correspondent à l'estimation de la seconde équation du modèle présentée en annexe de la version électronique de l'article. 
Toutefois, on peut s'interroger sur le sens de la causalité entre travail étudiant et abandon. Ainsi, la décision d'occuper un emploi durant les études est-elle la cause ou la conséquence de l'échec ? En effet, il est difficile, a priori, de savoir si c'est le fait d'occuper un emploi salarié qui conduit à se désintéresser des études ou si, au contraire, l'abandon résulte d'un désintérêt à l'égard des études qui incite à occuper un emploi en parallèle.

\section{Encadré 2. Travail salarié étudiant et abandon des études : cause ou conséquence?}

Afin d'essayer d'expliquer le sens de la causalité, nous avons réalisé deux estimations supplémentaires (1). La première est un modèle probit bivarié non récursif (l'indicatrice d'emploi salarié n'apparaît pas dans l'équation associée à l'abandon). Le coefficient de corrélation, positif (2), indique que des variables explicatives non observées dans les équations affectent dans le même sens les deux probabilités étudiées; par exemple, l'absence de motivation pour les études suivies (non observée dans l'enquête) augmente la probabilité d'abandonner son année mais aussi la probabilité d'occuper un emploi salarié. Pour savoir si l'abandon affecte l'emploi salarié ou l'inverse, nous avons réalisé l'estimation d'un modèle récursif avec pour première équation la probabilité d'abandonner ses études, pour seconde équation, la probabilité d'exercer un emploi salarié, et comme variable explicative, le fait d'avoir abandonné. Le coefficient associé à la variable abandon et le coefficient de corrélation ne sont pas significatifs

(1) : Les résultats de ces deux estimations sont disponibles auprès de l'auteur, sur demande.

(2) : Le signe du coefficient de corrélation est inversé par rapport au modèle présenté dans ce travail, car dans cette modélisation, l'emploi et l'abandon ne sont pas en même temps des variables explicatives.

Il semblerait que ce ne soit pas l'abandon des études qui suscite l'emploi salarié hors cursus mais plutôt l'inverse. Les étudiants qui abandonnent leurs études auraient une préférence pour le travail (Montmarquette \& al., 2007) ${ }^{15}$.

Pour revenir au Tableau 2, seules les caractéristiques déterminant les parcours scolaires (passé ou actuel) des étudiants et le genre ont un impact sur la décision d'abandon les études.

Les redoublements dans l'enseignement secondaire augmentent les chances d'abandonner son année. Toutefois, le retard scolaire sans redoublement de classe favorise une baisse de la probabilité d'abandonner ses études. Ainsi, il se pourrait que les étudiants déjà confrontés à l'échec scolaire accordent moins d'importance à leurs études et soient peut-être moins motivés. Le baccalauréat obtenu a aussi une forte influence sur la décision d'abandonner sa L1, puisque l'avoir obtenu avec mention diminue la probabilité d'abandonner sa L1 et

15. Des informations précises sur les dates de début de l'activité salariée et sur celles de l'abandon permettraient de mieux repérer le sens de la causalité. 
que les titulaires d'un baccalauréat littéraire ont plus de chances d'abandonner leur formation que les titulaires de baccalauréat scientifique.

Notons également que les hommes ont une probabilité plus élevée d'abandonner leurs études que les femmes. Mais nous n'observons aucun effet quant au lieu de naissance des parents, ni au fait qu'un des parents ait suivi des études supérieures. Nous avons considéré le niveau scolaire des parents comme étant une variable proxy de la catégorie socioprofessionnelle des parents, qui devrait motiver les étudiants à poursuivre leurs études par mimétisme. Toutefois, cette variable n'a pas d'impact sur l'abandon de la première année et cela peut se justifier par la définition que nous avons retenue de l'abandon. En effet, celle-ci englobe aussi bien les étudiants qui arrêtent définitivement leurs études que ceux qui les reprennent l'année suivante. Raison pour laquelle la non-significativité de la variable indiquant le niveau d'études des parents pourrait se comprendre.

De plus, le désintérêt pour la formation pourrait provenir du choix de la formation. D’une part, si la licence suivie n'est pas le premier choix de l'étudiant et, d'autre part, si l'étudiant souhaite entrer sur le marché du travail à l'issue d'une formation courte (bac +2 ou bac +3 ), la probabilité d'abandonner la L1 suivie est plus élevée. En revanche, la filière suivie n'a aucun impact sur les chances d'abandon.

De même, les caractéristiques subjectives impactent partiellement les chances d'abandon. En effet, la confiance en soi et la vision de son avenir n'ont aucun effet. Toutefois, avoir des problèmes personnels autres que financiers augmente la probabilité d'abandonner ses études.

Enfin, il semble ressortir un effet régional sur la probabilité d'abandon des études, puisque les étudiants habitant Lille semblent davantage abandonner leurs études que ceux vivant à Lyon. Il pourrait s'agir ici d'un effet des exigences académiques propres à chaque université et de leur modalité d'orientation ou d'accompagnement.

\subsection{L'emploi salarié, un effet négatif et significatif sur la réussite universitaire}

Cette estimation est présentée dans la troisième colonne ${ }^{16}$ du Tableau 2. Elle correspond à l'estimation de la seconde équation du modèle présenté en annexe de la version électronique de l'article.

Le travail salarié a un impact négatif et significatif sur la réussite universitaire. Ce résultat est conforme à ceux obtenus dans la littérature ( $c f$. Body, 2014 ; Body \& al., 2014) ou encore Beffy \& al. (2009 ; 2013). En effet, le temps destiné aux révisions pourrait être celui qui est occupé par l'emploi salarié, ce qui expliquerait l'impact négatif des emplois

16. Les résultats associés à l'équation de l'emploi, comparables à ceux présentés dans le Tableau 2 , ne sont pas reproduits pour les modèles présentés dans le paragraphes 4.2, mais sont disponibles sur demande. 
hors cursus sur la réussite universitaire. Lorsque l'on introduit, dans nos estimations, le type d'emploi ${ }^{17}$ (régulier ou temporaire, cf. Tableau 3), on observe que travailler régulièrement au cours de l'année universitaire pénalise plus fortement les chances de réussite et augmente la probabilité d'abandon des études, comme on peut l'observer dans la littérature (cf. Body, 2014 ; Body \& al., 2014) ${ }^{18}$.

Concernant les caractéristiques individuelles déterminant le parcours scolaire, nos résultats sont conformes à la littérature ( $c f$. Duguet $\&$ al., 2016, par exemple). Ainsi, le redoublement au lycée diminue les chances de réussir sa L1 ; la mention au baccalauréat ou encore la préparation d'un baccalauréat scientifique augmentent la probabilité de réussite.

Les caractéristiques liées à l'année universitaire influent la probabilité de réussite. D’une part, la filière suivie impacte les chances de valider l'année universitaire, puisque les étudiants inscrits en sciences humaines ou en lettres ont plus de chances de réussite que ceux inscrits en sciences fondamentales qui eux ont moins de chances de valider leur année que les étudiants en sciences sociales. La localisation de l'université semble avoir un impact sur la probabilité de réussite. Ainsi, étudier en région parisienne augmente les chances de valider son année, quand étudier à Lille les diminue. Ces variables de filières et de localisation peuvent être associées à la singularité des études en fonction de la ville et du cadre de vie, ainsi qu'aux exigences académiques propres aux universités et aux filières.

D'autre part, si la licence suivie n'est pas le premier choix d'études ou si les étudiants souhaitent suivre des études courtes (bac +2 ou bac +3 ), la probabilité de réussite est plus faible. Le manque de motivation pour les études suivies est un des éléments explicatifs de ce résultat.

Les caractéristiques individuelles et familiales agissent sur la réussite universitaire. D'une part, les hommes réussissent moins leur L1 que les femmes. D'autre part, le fait qu'un des parents ait suivi des études supérieures augmente la probabilité de réussite. Cette information est pour nous une variable proxy des origines sociales de l'étudiant. Ces dernières exerceraient une influence sur la motivation à poursuivre un cursus universitaire. Ce résultat pourrait donc être associé à une forme de reproduction sociale.

Enfin, les caractéristiques subjectives impactent partiellement les chances de réussite. En effet, seuls les étudiants ayant « des problèmes personnels non financiers » (cf. note 6) ont moins de chances de réussir leur année universitaire. Cependant, le manque de confiance en soi n'a aucun impact sur la probabilité de réussite.

17. La fréquence de l'emploi, considérée dans cet article, est déterminée par la nature des emplois exercés. Est considéré comme travail régulier tout emploi (CDI ou CDD) exercé de manière continue au cours de l'année universitaire. L’emploi occasionnel englobe donc l'ensemble d'activités rémunérées exercées de manière ponctuelle durant l'année universitaire.

18. On note que les coefficients de corrélation, significatifs et positifs, viennent atténuer ces effets. 
Tableau 3. Effet de la fréquence de l'emploi étudiant sur les résultats universitaires (*)

\begin{tabular}{|lcc|}
\hline \multicolumn{1}{|c|}{ Type de travail } & Coefficient & oEY \\
\hline Ensemble & $-0,855$ & 0,391 \\
\hline Occasionnel & $-0,366$ & \\
& & $0,210^{*}$ \\
\hline Régulier & $-0,999$ & \\
\hline
\end{tabular}

Lecture : pour chaque colonne, le chiffre présenté est le paramètre estimé significatif ( ${ }^{*}$ significatif à 10\%, N : modalités non significatives). Note de lecture : Toutes choses égales par ailleurs, le fait d'exercer un emploi salarié pendant ses études réduit significativement la probabilité de réussir ses études. Avec ce type de modélisation, on peut juste juger du sens de l'impact et non de l'ampleur de la probabilité.

$\left(^{*}\right)$ : Les résultats relatifs aux autres variables explicatives ont été omis pour alléger le texte et restent disponibles auprès de l'auteur. Source : Panel 1995 , ministère de l'Éducation nationale.

\section{Conclusion}

Afin d'éclairer le débat relatif aux causes du taux élevé d'échec survenant en fin de première année d'université, en France, cet article vise à examiner le lien entre l'activité professionnelle et les résultats universitaires des néo-bacheliers inscrits en première année de Licence. La particularité de cette analyse est d'étudier exclusivement le cas des L1 en France. La plupart des articles empiriques ne tiennent pas compte de la L1 en raison du manque d'informations précises sur cette première année.

Pour ce faire, nous avons entrepris, dans un premier temps, de déterminer les différentes caractéristiques qui ont un impact sur la décision d'occuper un emploi hors cursus durant l'année universitaire et ensuite, de relever les conséquences de cette activité salariée sur les résultats universitaires (abandon, validation ou non de l'année universitaire).

Ainsi, le genre, la scolarité passée, la filière suivie, le type de logement, l'aide financière familiale, l'obtention d'une bourse d'études, la région de résidence, tout comme certaines caractéristiques subjectives (vision de l'avenir et problèmes personnels non financiers) constituent les éléments qui interagissent sur le choix d'exercer un emploi salarié.

De plus, les activités salariées hors cursus ont un impact négatif sur la validation de la première année de licence et favorisent l'abandon des études. De surcroît, une activité salariée exercée régulièrement durant l'année est très pénalisante. Ces résultats confirment ce qui a été jusque-là relevé dans la littérature pour des étudiants de niveau supérieur.

Afin de diminuer le taux d'abandon et augmenter le taux de réussite, plusieurs mesures de politiques publiques sont envisageables. Les emplois du temps pourraient ainsi être aménagés (travaux dirigés le soir ou le samedi, utilisation des TICE ${ }^{19}$ ). Le nombre hebdomadaire d'heures travaillées durant l'année universitaire gagnerait à être limité. Cette perte de revenu pour les étudiants salariés pourrait être compensée par des aides financières, et

19. Technologies de l'Information et de la Communication pour l'Enseignement. 
ainsi diminuer la probabilité d'occuper un emploi salarié. Faciliter l'accès au crédit, par la mise en place de prêt à remboursement conditionnel (comme le suggèrent Grégoir, 2008 ; Courtioux, 2009 ; 2010 ; Harari-Kermadec \& Moulin, 2015), pourrait aussi représenter une alternative. Enfin, l'institution pourrait mettre à disposition des étudiants les ressources qu'elle juge nécessaires et veiller à ce qu'elles soient accessibles à ceux qui en ont le plus besoin (David \& Melnik, 2014).

Cette étude permet de repérer un effet moyen de l'emploi salarié. Un prolongement pertinent de ce travail serait d'étudier la sensibilité de l'effet de l'emploi salarié sur des souséchantillons d'étudiants (sexe, type de baccalauréat, origines sociales ...). Une telle étude permettrait peut-être de mieux cibler les étudiants afin de diminuer le recours à l'emploi salarié.

Afin de mesurer de manière plus fine l'effet de l'activité, un autre prolongement de ce travail pourrait être la prise en compte de l'intensité de l'activité salariée en termes d'heures hebdomadaires travaillées ou de la catégorie de l'activité occupée (en lien ou non avec les études).

Toutefois, nos résultats révèlent que le travail salarié n'est pas le seul élément qui impacte le taux d'échec en première année (ce taux englobant l'abandon et la non-validation de l'année universitaire). Une mauvaise orientation, un choix par défaut ou encore une difficulté à s'adapter dans un contexte très différent du lycée semblent être également des facteurs explicatifs de ce taux d'échec, tout comme la non-convergence entre les objectifs individuels et ceux de l'institution (David \& Melnik, ibid.). Par conséquent, cet échec interroge les processus de sélection et d'accompagnement des futurs bacheliers dans le choix de leur orientation.

\section{Bibliographie}

Baum S. (2010), "Understanding the Working College Student: New Research and Its Implications for Policy and Practice", chapter Undergraduate Work and the Student Aid System, Stylus.

Beaud S. (2008), "Enseignement supérieur : la "démocratisation scolaire" en panne", Formation Emploi, 101, pp. 149-165.

Beaupère N. \& Boudesseul G. (2009), “ Sortir sans diplôme de l'université : comprendre les parcours d'étudiants décrocheurs”, La Documentation française.

Beffy M., Fougere D., Maurel A. (2009), “L’impact du travail étudiant salarié sur la réussite et la poursuite des études universitaires ”, Economie et Statistique, 422, pp. 31-50.

Beffy M., Fougère D., Maurel A. (2013), "The effect of college employment on graduation: evidence from France", CEPR Discussion Paper, 9565. 
Belghith F. \& Vourc'h R. (2011), "Eurostudent IV : une comparaison européenne des conditions de vie des étudiants", Rapport technique, OVE.

Belghith F. (2015), L'activité rémunérée des étudiants. Une diversité de situations aux effets contrastés, Rapport technique, OVE.

Bisseret N. (1968), “La «naissance» et le diplôme : Les processus de sélection au début des études universitaires”, Revue française de sociologie, pp. 185-207.

Bodin R. (2009), “Les signes de l'élection ”, Actes de la recherche en sciences sociales, (3), pp. 80-87.

Bodin R. \& Millet M. (2012), “L'université, un espace de régulation” , Sociologie, 2(3), pp. 225-242.

Body K. M.-D. (2014), Étude micro-économétrique de l'impact du travail salarié étudiant sur la réussite à l'université, Thèse de doctorat es sciences économiques, université de Poitiers.

Body K. M.-D., Bonnal L. \& Giret J.-F. (2014), “Does student employment really impact academic achievement? The case of France”, Applied Economics, 46(25), pp. 3061-3073.

Brint S. \& Cantwell A. M. (2010), "Undergraduate time use and academic outcomes: Results from the university of California undergraduate experience survey 2006", Teachers College Record, 112(9), pp. 2441-2470.

Coulon A. (1997), " Le métier d'étudiant : l'entrée dans la vie universitaire ”, Paris, Economica-Anthropos.

Courtiaux P. (2010), “ L'effet du système socio-fiscal sur les rendements privés de l'enseingement supérieur. Une analyse par microsimulation ”, Positions Papers, IDHEC.

David S. \& Melnik-Olive E. (2014), “Le décrochage à l'université, un processus d'ajustement progressif ? ”, Formation Emploi, 128, pp. 81-100.

Duguet A., Le Mener M. \& Morlaix S. (2016), "The Key Predictors of Success in University in France: What Are the Contributing Factors and Possible New Directions in Educational Research?”, International Journal of Higher Education, 5 (3), pp. 222-235.

Ehrenberg R. G. \& Sherman D. R. (1987), "Employment while in college, academic achievement and postcollege outcomes", Journal of Human Resources, 22(1), pp. 1-24.

Froment B. (2012), "Les effets du travail salarié en première année universitaire ", SociologieS [En ligne], Premiers textes, mis en ligne le 09 mai 2012, consulté le 13 octobre 2016. URL : http://sociologies.revues.org/4006

Galland O. (2016), "Le budget étudiant", in Giret J.-F., Van de Velde C. \& Verley E. (dir.), Les vies étudiantes. Tendances et inégalités, Paris, La Documentation française. 
Gregoir S. (2008), “ Les prêts étudiants peuvent-ils être un outil de progrès social ? ”, Positions Papers, IDHEC.

Gruel L. (2002), “ Les conditions de réussite dans l'enseignement supérieur ”, OVE infos, 2, pp. 1-7.

Harari-Kermadec H. \& Moulin L. (2015), “ Postface - De la mise en concurrence à la mise en marché de l'enseignement supérieur”, Formation Emploi, 132.

Hochschild A. (1983), “The Managed Heart”, Berkele, University of California Press.

Ichino A., Mealli F. \& Nannicini T. (2008), "From temporary help jobs to permanent employment: What can we learn from matching estimators and their sensitivity?", Journal of Applied Econometrics, 23(3), pp. 305-327.

Kalenkoski C. M. \& Pabilonia S. W. (2009), "Does Working While in High School Reduce US Study Time?”, Social Indicators Research, 93(1), pp. 117-121.

Lafontaine A. \& Terwagne S. (1993), Les conditions d'accès à l'enseignement supérieur dans la Communauté européenne, Paris, Unité européenne d'EURYDICE de la CCE.

Mcneal Jr R. B. (1997), "Are students being pulled out of high school? The effect of adolescent employment on dropping out”, Sociology of Education, pp. 206-220.

MESR-SIES (2013), “ Réussite et échec en $1^{\mathrm{er}}$ cycle ”, Note d'information Enseignement Supérieur et Recherche 13.10, novembre.

Michaut C. \& Romainville M. (Éd.) (2012), Réussite, échec et abandon dans l'enseignement supérieur, Bruxelles, De Boeck, pp. 53-68.

Montmarquette C., Viennot-Briot N. \& Dagenais M. (2007), "Dropout, school performance, and working while in school", The Review of Economics and Statistics, 89(4), pp. $752-760$.

Morlaix S. \& Suchaut B. (2015), “The social, educational and cognitive factors of success in the first year of university: A case study”, International Review of Education, pp. 1-22.

Romainville M. (1997), “ Peut-on prédire la réussite d'une première année universitaire ?", Revue Française de Pédagogie, vol. 119, pp. 81-90.

Scott-Clayton J. (2012), "What explains trends in labor supply among U.S. undergraduates, 1970-2009?”, Working Paper 17744, National Bureau of Economic Research.

Verley E. \& Zilloniz S. (2010), “L’enseignement supérieur en France : un espace segmenté qui limite l'égalisation des chances", Formation Emploi, 110, pp. 5-18.

Wharton A. S. (2004), "Femmes, travail et émotions : concilier emploi et vie de famille", Travailler (2), pp. 135-160. 